\title{
Anti-tumor effects of LncRNA-CR594175 silenceing on hepatocellular carcinoma cell line HepG2 in vivo and in vitro
}

\section{Quan Liu}

The Fifth Affiliated Hospital of Zhengzhou University

\section{Xuxu Yu}

The First Affiliated Hospital of Zhengzhou University

\section{Minjie Yang}

The First Affiiated Hospital of Zhengzhou University

\section{Xiangke Li}

The First Affiliated Hospital of Zhengzhou University

\section{Xuejia Zhai}

The First Affiliated Hospital of Zhengzhou University

\section{Yujin Lian}

The First Affiliated Hospital of Zhengzhou University

\section{Zhong Chen}

The First Affiliated Hospital of Zhengzhou University

\section{Qingxia Fan}

The First Affiliated Hospital of Zhengzhou University

\section{Lijie Song ( $\square$ culleen@163.com)}

The First Affiliated Hospital of Zhengzhou University https://orcid.org/0000-0003-0102-2407

\section{Wecai Li}

The First Affiliated Hospital of Zhengzhou University

\section{Research}

Keywords: CR594175, Hepatocellular Carcinoma, Wnt Signaling Pathway, hsa-miR142-3p, proliferation, invasion, HepG2

Posted Date: February 19th, 2020

DOl: https://doi.org/10.21203/rs.2.23988/v1

License: (c) (1) This work is licensed under a Creative Commons Attribution 4.0 International License. Read Full License 


\section{Abstract}

Background Hepatocellular carcinoma (HCC) is one of the cancers of highest incidence and mortality worldwide.

Methods High-throughput screening was applied to find the most varied expressed LncRNA in primary hepatocellular carcinoma and explore the interaction network of it.

Results We found that IncRNA-CR594175 was the IncRNA of most varied expression, which was lower in adjacent noncancerous tissues than in primary HCC, and was lower in primary HCC than in its metastases. Knockdown of CR594175 inhibited the proliferation and invasion of HCC cells HepG2. The mechanism study revealed that CR594175, as a RNA sponge, broke the negative regulation of CTNNB1 (Catenin, beta-1) by hsa-miR142-3p, and once CR594175 was silenced, the highly expressed hsa-miR142$3 p$ regained its regulation on CTNNB1 and inhibited the proliferation and invasion through Wnt signaling pathway.

Conclusions Our present study demonstrates for the first time that CR594175 silencing suppressed proliferation and invasion of HCC cells HepG2 in vivo and in vitro by restoring the negative regulation of CTNNB1 by hsa-miR142-3p, laying a solid theoretic base for using IncRNA-CR594175 as genetic therapy target for HCC and offering a reasonable explanation for inactivation of miRNA in difference tumors or tumor of different stages.

\section{Background}

Hepatocellular carcinoma (HCC) is one of the cancers of highest incidence and mortality worldwide [1], featuring a low complete resection rate and a high postoperative recurrence rate [2-3], which is mainly driven by high invasiveness and intrahepatic and/or extrahepatic metastasis [4]. Therefore, the study on the mechanism for HCC cells proliferation and invasion is of great significance to improving the prognosis of patients with $\mathrm{HCC}$ and to lengthening the survival time. At present, surgery combined with pre- and post-operative chemotherapy is the mainstay of treatment for HCC but this traditional therapeutic method doesn't work for postoperative recurrence and metastasis. So, in recent years, searching for new therapeutic target of gene treatment for HCC never stopped.

Both miRNAs and IncRNAs play important roles in regulating cellular processes [5-7]. CR594175 is the IncRNA of the most differential expression among HCC, para-cancerous tissue and metastases we found through screening and sequencing of the transcriptome, and the expression of CR594175 was found to decrease from metastases to primary HCC and then to adjacent non-cancer tissues, which suggests that CR594175 may be involved in proliferation and invasion of HCC. According to our screening data, CTNNB1 was highly correlated with the process of HCC. In our study, we found that CTNNB1 was highly expressed in HCC cell line HepG2, and CR594175 silencing reduced CTNNB1 protein levels (but not mRNA levels). The evident post-transcriptional regulation hinted that CR594175 may influence proliferation and invasion in HCC cells through regulating the expression of CTNNB1 and the link may be miRNA, a typical 
post-transcriptional regulation way, given that RIP experiment proved that CR594175 did not bind to CTNNB1 protein directly. We searched for a miRNAs having potential to bind to 3'-UTR (Untranslated Regions) of CTNNB1 as well as the ones that theoretically bind to CR594175, and found hsa-miR142-3p, which has one theoretical binding site on 3'-UTR of CTNNB1 and two sites on CR594175. Therefore, we think that as a miRNA sponge, CR594175 may impair the negative regulation of CTNNB1 by hsa-miR142$3 p$, since hsa-miR142-3p levels in primary and metastatic HCC is higher than in adjacent non-cancer tissues, and in metastatic HCC is higher than in primary HCC, that is, hsa-miR142-3p loses its ability to suppress proliferation and invasion of HCC cells through negatively regulating CTNNB1, due to high CR594175 expression and competitive binding to hsa-miR142-3p with 3'UTR of CTNNB1 may contribute to its inactivation.

The pathogenesis of $\mathrm{HCC}$ is complicated, in which Wnt/ $\beta$-catenin signaling pathway play an important role and become a hotspot in the study of HCC. According to medical basic research, Wnt/ $\beta$-catenin signaling pathway can affect the process of HCC mainly by expression regulation of downstream genes and proteins. As the key of $\mathrm{Wnt} / \beta$-catenin signaling pathway, increasing expression of $\beta$-catenin indicates activation, which makes it a critical gene in the research of HCC regulation. The inhibitory effects of Wnt signaling pathway by nonsteroidal anti-inflammatory drugs and valproic acid has been implied in clinical adjuvant therapy of HCC, nevertheless, the inhibitor of $\beta$-catenin becomes the new study direction in prevent precancerous lesions such as hepatitis and liver cirrhosis from deteriorating. It has already been verified that R-Etodolac, as the inhibitor of $\beta$-catenin, can suppress proliferation of HCC cell lines HepG2 and Hep3B effectively [8]. In addition to this, a variety of miRNAs has been confirmed to have an influence on the process of HCC through the regulation of Wnt/ $\beta$-catenin signaling pathway [9-12] and the targets are different. It's uncommon for miRNA to involve Wnt signaling pathway targeting $\beta$-catenin until now, but our study revealed miRNA-has-miR142-3p has a negative regulation on the progress of HCC by $\beta$-catenin. The data indicated that increased expression level of hsa-miR142-3p from HCC metastasis to primary lesion and then to para-cancerous tissues, but it's puzzling that the expression of $\beta$-catenin protein remains stable. So, it's crucial for the theoretical research and clinical treatment to find the reason why antineoplastic miRNA like has-miR142-3p inactivated in the process of HCC. The study on the interaction between miRNAs and IncRNAs will revolutionize our knowledge about cell structural network and regulatory network, and bring in immeasurable scientific and clinical value.

\section{Materials And Methods}

\section{Cell culture}

HCC cell lines HepG2, obtained from the Cell Bank of the Chinese Academy of Sciences (Shanghai, China), were maintained in RPMI-1640 (Invitrogen, CA, USA) supplemented with 10\% FBS (Fetal bovine calf serum, Invitrogen). 293TN cells, purchased from ATCC (MD, USA), were maintained in DMEM (Dulbecco minimum essential medium, Invitrogen) supplemented with $10 \%$ FBS. All these adherent cells were passaged by $0.25 \%$ trypsin digestion (Invitrogen) and incubated in an atmosphere of $5 \% \mathrm{CO}_{2}$ at 37 ${ }^{\circ} \mathrm{C}$. 


\section{Assessment of CR594175, hsa-miR142-3p, CTNNB1 protein and Wnt pathway related proteins in HCC tumors and its metastatic tissues}

HCC, the metastatic tissues and para-carcinoma tissues from 24 patients (diagnosed in the First Affiliated Hospital of Zhengzhou University and detailed patient information was shown in Tab.1) were collected, followed by total RNA extraction and real-time PCR for measurement of CR594175 and hsa-miR142-3p and total protein extraction and western blotting for CTNNB1 and Wnt pathway related proteins (Ecadherin, C-myc, CyclinD1 and MMP-9).

\section{Lentivirus packaging}

A siRNA sequence complementarily binding to CR594175 was chosen. The target sequences of siRNA (5'GAATCCTCGGAGACAGCAG-3') are homologous to CR594175, respectively. The oligonucleotide templates of these shRNAs were chemically synthesized and cloned into the linear pSIH1-H1-copGFP shRNA Vector (System Biosciences, CA, USA) which was obtained through digestion by BamH I and EcoR I(Takara, Dalian, China) and purification by agarose gel electrophoresis. An invalid siRNA sequence (5'AATCGTCGAGGGCCAGACA-3') was used as a NC (negative control). Sequencing was used to confirm the vectors constructed ( $\mathrm{pSIH1}$-shRNA-CR594175 and pSIH1-NC). The CDS sequence of human CTNNB1 (NM_001904.3) was amplified by using the primers 5'-GGAATTCGCCACCATGGCTACTCAAGCTGATTTG-3' and 5'-CGGGATCC TTACAGGTCAGTATCAAACC-3', which contain an EcoRI cutting site and Kozak sequence and a Bamhl cutting site, respectively, with the CDNA prepared by reverse transcription of RNA isolated from 293TN cells. The PCR product was digested and cloned into PcDH1-CMV lentiviral expressing vector; the recombinant vector was named pcDH1-CTNNB1. The products of the vectors were confirmed by DNA sequencing. Endotoxin free DNA was prepared in all cases.

One day before transfection, 293TN cells were seeded into 10-cm dishes(Corning,NY,USA). $2 \mu \mathrm{g}$ of each pSIH1-shRNA-CR594175 vector or pSIH1-NC and $10 \mu \mathrm{g}$ pPACK Packaging Plasmid Mix (System Biosciences) were co-transfected using Lipofectamine 2000(Invitrogen) in accordance with the manufacturer's protocol. The medium was replaced with DMEM plus $1 \%$ FBS. Forty eight hours later, the supernatant was harvested and then cleared by centrifugation at $5000 \times \mathrm{g}$ at $4{ }^{\circ} \mathrm{C}$ for 5 minutes, and passed through a $0.45 \mu \mathrm{m}$ PVDF membrane (Millipore, MI, USA). The titer of virus was determined by gradient dilution. The packaged lentiviruses were named as Lv-shRNA-CR594175 and Lv-NC. Recombinant lentivirus Lv-CTNNB1 and Lv-miR142-3p was packaged by the same way.

\section{Genetic intervention through a lentiviral approach}

Cells were divided into four groups: a control group, Lv-NC group (infected with Lv-NC), Lv-shRNACR594175 group (infected with Lv-shRNA-CR594175) and Lv-CTNNB1 group (infected with Lv- CTNNB1). HepG2 in logarithmic phase were seeded to 6-well plates at $5 \times 105$ cells/well. One day later, viral solution was added at an $\mathrm{MOI}$ of 10 . The infection efficiency was evaluated by observing and analyzing the fluorescent mark 72 hours after infection. And total RNA and protein were isolated from the cells and subjected to real-time PCR and western blotting for CR594175 and CTNNB1 protein, respectively. 


\section{Luciferase experiment}

Total RNA was extracted from HepG2, reverse-transcribed into CDNA, and $2 \mu \mathrm{l}$ of the reaction product subsequently used as a template for PCR. Primers were designed that targeted the 3'-UTR of the CTNNB1 gene such that flanking Xbal restriction sites were introduced into the $127 \mathrm{bp}$ (base-pair) PCR product containing the 5'-AACACTA-3' hsa-miR142-3p target site. The forward and reverse primer sequences were 5'- GCTCTAGATTAAGAATTGAGTAATGG-3' and 5'-GCTCTAGA ACTAATTGGACCATTTTC-3', respectively. PCR reaction conditions were as follows: 35 cycles of a $94{ }^{\circ} \mathrm{C}$ denaturing step for $30 \mathrm{~s}$, a $55^{\circ} \mathrm{C}$ annealing step for $30 \mathrm{~s}$, and a $72{ }^{\circ} \mathrm{C}$ elongation step for $10 \mathrm{~s}$. The PCR product was digested with Xbal(Takara)and cloned into the pGL3-promoter luciferase reporter vector (Promega, MI,USA) to generate the vector pGL3wt-CTNNB1. The hsa-miR142-3p target site in the pGL3-WT-CTNNB1 vector was mutated from 5'AACACTA -3' to 5'- CATAACA -3' to construct the mutated reporter vector, pGL3-mt-CTNNB1. The products of all cloning and mutagenesis reactions were confirmed by DNA sequencing. Endotoxin free DNA was prepared in all cases. The hsa-miR142-3p mimic(5'-UGUAGUGUUUCCUACUUUAUGGAtt-3'), the hsamiR142-3p inhibitor(5'- UCCAUAAAGUAGGAAACACUACAtt-3), and negative control miRNA (NC,5'UGUAGUGUUUCCUACUUUAUGGAtt-3') were all chemically synthesized(Invitrogen).

We used Targetscan (http://www.targetscan.org/) to predict whether a hsa-miR142-3p binding site exists within the 3'-UTR of human CTNNB1 mRNA. The results showed that a seven-base hsa-miR142-3p seed sequence is present in the 3'-UTR of CTNNB1 mRNA. The same tool was used to predict the binding sites of hsa-miR142-3p on CR594175.A suspension of 293TN cells in logarithmic phase growth was prepared and the number of viable cells counted using a hemocytometer in conjunction with trypan blue staining. The cells were seeded into six-well plates at a concentration of $2 \times 10^{5}$ cells per well and maintained in Dulbecco's Modified Eagle's medium supplemented with $10 \%$ fetal calf serum at $37^{\circ} \mathrm{C}$ for 24 hours in a 5

$\% \mathrm{CO} 2$ atmosphere. The transfection of plasmid DNA and RNA was performed using Lipofectamine 2000 (Invitrogen, USA). Transfection of cells with pRL-TK(100 ng) served as a reference for luciferase detection. Luciferase activity was measured using the dual luciferase reporter assay system (Promega, USA) 48 hours after transfection. The experiment to observe the effect of CR594175 depletion on the inhibition of luciferase by hsa-miR142-3p mimics was carried out in HepG2 cells, the plasmid transfection and luciferase activity assay the same as used in validation of target site.

\section{Cellular proliferation assay}

HepG2 cells were divided into seven groups: a control group, Lv-NC group, Lv-shRNA-CR594175 group, LvmiR142-3p group, Lv-shRNA-CR594175 and Lv-miR142-3p group, Lv-CTNNB1 group, and Lv-shRNACR594175 and Lv-CTNNB1 group. Fourty-eight hours after infection, HepG2 cells groups were trypsinized, and seeded into 96 -well plates at a density of $1 \times 10^{4}$ cells per well. The cells were cultured under normal conditions and cell viability was examined using CCK-8 at 24-, 48-, and 72-hour time points. Briefly, 10 uL CCK-8 solution (Dojindo, Japan) was added, and the cells then cultured under normal conditions for an additional 4 hours before measurement of absorbance at $490 \mathrm{~nm}$. 


\section{Cell invasion assay}

HepG2 cells infected with recombinant lentiviruses 72 hours, trypsinized and used for invasion assay. Cell invasion experiments were performed using the QCMTM 24-well Fluorimetric Cell Invasion Assay kit (ECM554, Chemicon International, USA) according to the manufacturer's instructions. The kit uses an insert polycarbonate membrane with an 8- $\mu \mathrm{m}$ pore size. The insert was coated with a thin layer of EC Matrix $^{\mathrm{TM}}$ that occluded the membrane pores and blocked migration of non-invasive cells. Culture medium $(500 \mu \mathrm{l})$ supplemented with $10 \%$ FBS was used as chemoattractant. Cells that migrated and invaded the underside of the membrane were fixed in $4 \%$ paraformaldehyde. The invading cells were stained by Calcein-AM, and the number was then determined by fluorescence and reported as relative fluorescence units (RFUs). The grouping and cell treatment were the same as those for cell viability assay. Seventy-two hours after lentiviral infection, cells were trypsinized and the viable cells were counted by using trypan blue staining, and seeded into the upper chamber of transwell at a density of $5 \times 10^{5} \mathrm{cells} / \mathrm{well}$, and incubated under normal conditions for 48 hours, and then stained and counted. The grouping was the same as in the proliferation assay.

\section{Effect of CR594175 silencing on expression of $\beta$-catenin and downstream functional proteins of Wnt pathway}

HepG2 cells were divided into three groups: a control group, Lv-NC group and Lv-shRNA-CR594175. Cells in logarithmic phase were seeded to 6-well plates at 5×105 cells/well. One day later, viral solution was added and the infection efficiency was evaluated by observing and analyzing the fluorescent mark 72 hours after infection. Protein were isolated and subjected to western blotting for CTNNB1, E-cadherin, Cmyc, CyclinD1 and MMP-9 protein, respectively.

\section{Real-Time PCR}

Total RNA was isolated with Trizol Reagent (Invitrogen) according to the manufacturer's instruction and reversely transcribed into cDNA using M-MLV Reverse Transcriptase (Takara, Japan) and oligo(dT)18 primer (Takara). The following specific primers were used in quantitative PCR of human CR594175 and $\beta$ actin: CR594175: 5'-ACATATAATTGAATATTATT-3' and 5'-TTAGCCATTTTAAAATGTATGG-3'; and $\beta$-actin: 5'CCTGTACGCCAACACAGTGC-3' and 5'-ATACTCCTGCTTGCTGATCC-3'. The length of amplified products were $321 \mathrm{bp}$ and $211 \mathrm{bp}$, respectively. Real-time PCR was performed using SYBR Premix Ex Taq ${ }^{\text {TM }} \mathrm{kit}^{\mathrm{d}}$ and TP800 System (Takara BIO). cDNA from $200 \mathrm{ng}$ total RNA was used as the template. The PCR reactions was carried out under the following conditions: 40 cycles of denaturation at $95^{\circ} \mathrm{C}$ for $10 \mathrm{~s}$, annealing at $60^{\circ} \mathrm{C}$ for $20 \mathrm{~s}$ and extension at $72^{\circ} \mathrm{C}$ for $20 \mathrm{~s}$. The mRNA levels of CTNNB1 were normalized using the delta-delta $\mathrm{Ct}$ method, to the expression of an endogenous housekeeping gene, $\beta$-actin. The expression of hsa-miR142-3p was analyzed with the $2^{-\Delta \Delta \mathrm{Ct}}$ method. For each sample, triplicate determinations were performed, and mean values were adopted for further calculations. All values were normalized to an endogenous U6 control. The PCR primers for mature hsa-miR142-3p or U6 were designed as follows: hsa- 
miR142-3p sense: 5'- TGTAGTGTTTCCTACTTTATGGA-3' and reverse: 5'-GTCGTATCCAGTGCGTGTCGTG3'; U6 sense: 5'-GTGCTCGCTTCGGCAGCACAT-3' and reverse: 5'-TACCTTGCGAAGTGCTTAAAC-3'.

\section{Detection of protein contents in cell or tissues}

The total protein was extracted from the cells using M-PER mammalian protein extraction reagent (Pierce, IL, USA) or from tissues using T-PER tissue protein extraction reagent (Pierce). Equal amounts of protein ( $25 \mu \mathrm{g}$ per lane) estimated by a bicinchoninic acid (BCA) protein assay kit (Pierce) were loaded onto (11\%) SDS-PAGE gels and transferred onto nitrocellulose membranes. The blots were probed with a monoclonal antibody against human CTNNB1 (1:400), E-cadherin (1:200), C-myc (1:300), CyclinD1 (1:400) هMMP-9 (1:250) and $\beta$-actin (1:1000) (Santa Cruz, USA), followed the secondary HRP-conjugated anti-mouse/rabbit antibody (Abcam, CA, USA). After washing, the bands were detected by chemiluminescence and imaged with X-ray films. $\beta$-actin was used as an endogenous reference for normalization.

\section{Animal xenografts}

Nude mice were purchased from Shanghai SLAC Laboratory Animal Co.,Ltd (Shanghai, China) and housed at the animal experiment center of Zhengzhou University, where the implantation experiment was performed. All the protocols were previously approved by the Zhengzhou University Animal Ethics Committee. HepG2 cells $\left(1 \times 10^{6}\right)$ were suspended in $200 \mu$ medium, and injected subcutaneously into the flank regions of 48 female athymic nude mice. Two weeks after inoculation, visible subcutaneous tumors were detected, and the tumors were measured approximately $2.5 \mathrm{~mm}$ in diameter 3 weeks after inoculation. All animals were randomly divided into 3 groups ( 8 mice per group): the Model group, the NC group, and the CR594175-silencing group. For the intervention groups, each animal received $30 \mu \mathrm{l}$ recombinant lentivirus $\left(1 \times 10^{8} \mathrm{IFU}\right)$ twice a week since the second week for 4 weeks, while the model group received the same volume of saline instead. Tumor diameter was measured weekly since the second week, and the data was used to plot the tumor growth curves. The formula for calculating the tumor volume was: $\mathrm{V}=\mathrm{ab} 2 / 2, \mathrm{a}$ and $\mathrm{b}$ are the long and short diameters of the tumor, respectively.

\section{Statistical analysis}

All data is expressed as mean \pm SD, and analyzed by one way ANOVA. Least Significant Difference (LSD) was used for multiple comparisons between any two means. $P$-values $<0.05$ were considered statistically significant. All statistical analysis was performed using SPSS 13.0 software.

\section{Results}

Assessment of CR594175, hsa-miR142-3p and CTNNB1 protein in tissues from HCC and metastatic tissues 
The data of CTNNB1 mRNA and protein levels demonstrates that in comparison with para-carcinoma tissues, CTNNB1 protein was increased in HCC and its metastasis $(p<0.01)$, and more in HCC metastasis than in primary $\mathrm{HCC}(p<0.05)$; but there were no obvious differences between the mRNA levels in three groups of tissues $(p>0.05)$. These results suggest that the high expression of CTNNB1 is due to inactivation of post-transcriptional regulation. The expression of CR594175 and hsa-miR142-3p in the adjacent tissues, $\mathrm{HCC}$ and their metastasis were positively correlated with snail protein levels and was higher in the HCC and their metastasis than that in the adjacent tissues $(p<0.05)$ (Fig1-A). We also detected the downstream functional proteins of Wnt pathway E-cadherin, C-myc, CyclinD1 and MMP-9 levels in $\mathrm{HCC}$ and their metastasis as compared with the adjacent tissue $(p<0.01)$, that being more significant in $\mathrm{HCC}$ metastasis than in primary HCC $(p<0.05)$. A reverse trend was observed in E-cadherin to the three proteins mentioned above in these tissues (Fig1-B).

\section{Effect of silencing CR594175 and expression CTNNB1 via lentiviral approach HCC cells}

Recombinant lentiviruses, Lv-NC,Lv-shRNA-CR594175 and Lv-CTNNB1, were used to infect HepG2. GFP(Green fluorescent protein) was detected in most of the cells 72 hours after infection, and the proportion of GFP-expressing cells suggested that the gene delivery efficiency was higher than $95 \%$ in the HepG2 (Fig.2A). CR594175 was significantly decreased by Lv-shRNA-CR594175 ( $p<0.05)$, and no change in cells infected with Lv-CTNNB1( $p \otimes 0.05)$; CTNNB1 protein level was significantly increased by LvCTNNB1 and decreased by Lv-shRNA-CR594175 ( $p<0.05$ )(Fig.2B). These findings suggest that CR594175 silencing down-regulated CTNNB1 expression in HepG2, overexpression of CTNNB1 had no obvious effect on CR594175, so there is an obvious upstream and downstream relation between CR594175, hsamiR142-3p and CTNNB1.

\section{Luciferase experiments}

Our bioinformatics analysis identified an 8-base hsa-miR142-3p seed sequence in the 3'-UTR of CTNNB1 mRNA. We therefore constructed luciferase reporter vectors to verify whether this site represents a valid hsa-miR142-3p target. Reporter vectors were generated that contained the wild-type CTNNB1 3'-UTR or a variant in which the hsa-miR142-3p target site within the 3'-UTR had been mutated. Both reporter constructs expressed luciferase at a high level. However, the miR142-3p mimic significantly inhibited luciferase activity in cells transfected with the reporter vector encoding the wild type 3 '-UTR $(42.15 \pm 3.98$ vs. $8.07 \pm 0.88 ; p<0.01)$, while the miR142-3p inhibitor significantly increased luciferase activity in these cells $(43.02 \pm 2.02$ vs. $52.81 \pm 9.04 ; p<0.05)$. Conversely, in cells transfected with the reporter vector encoding the mutated hsa-miR142-3p target site, neither the miR142-3p mimic nor the miR142-3p inhibitor had any observable effect on luciferase activity $(p>0.05)$. Co-transfection of miR142-3p-NC (non-targeting control) had no effect on the luciferase activity of either of the vectors $(p>0.05)$. These results verified the presence of a hsa-miR142-3p target site in the 3'-UTR of CTNNB1 mRNA and demonstrated that binding of hsa-miR142-3p to this target site down-regulates CTNNB1 expression (Fig. $3 A)$. Interestingly, miR142-3p mimics lost its inhibition on the activity of luciferase expressed by wild-type 
(wt) luciferase reporter vector in HepG2, and regained the inhibition after CR594175 silencing (Fig. 3B). Taken together, these data suggest that CR594175 overexpression impaired negative regulation of CTNNB1 by hsa-miR142-3p.

\section{Effect of CR594175 knock down on proliferation and invasion of HepG2 cells}

As suggested by cell proliferation assays, CR594175 silencing effectively inhibited the proliferation of HepG2 cells 72 hours after infection $(p<0.01)$, while CTNNB1 overexpression could promote the proliferation of HepG2 cells $(p<0.05)$, and CTNNB1 overexpression could reverse the proliferation suppression caused by CR594175 silencing $(p<0.05)$. Overexpression of miR142-3p had no obvious effect on the proliferation of HepG2, but it showed a strong inhibitory activity of proliferation in the case of CR594175 silenced(Fig. 4A). Therefore, it was suggested that CR594175 silence inhibited cell proliferation by suppressing the expression of CTNNB1, which was reversed by exogenous expression of CTNNB1.Furthermore, cell invasion assay results demonstrated that CR594175 silencing inhibited invasion in HepG2 cells $(p<0.01)$, and CTNNB1 overexpression could reverse the invasion inhibition caused by CR594175 silencing $(P<0.05)$. Overexpression of miR142-3p had no obvious effect on invasion of HepG2, but it showed a strong inhibitory activity of invasion in the case of CR594175 silenced (Fig. 4B). Because exogenous CTNNB1 has no wild promoter, we deduced that some transcription factors may bind to CTNNB1 promoter and activate its transcription, which may mediate the regulation of CTNNB1 by CR594175. In vivo experiment showed that 4 consecutive weeks of CR594175 silencing significantly decreased tumor volume. After administration for five weeks, the tumor volume of the model group was $701.21 \pm 54.13 \mathrm{~mm}^{3}$, the NC control group was $672.34 \pm 49.06 \mathrm{~mm}^{3}$ and the CR594175 silencing group was $212.31 \pm 57.71 \mathrm{~mm}^{3}$. The tumor inhibition rates in the NC group and CR594175 silencing group were $4.12 \%$ and $69.73 \%$, respectively, with a statistically significant difference between the CR594175 silencing group and the other two groups $(p<0.01)$ (Fig. 4C).

\section{Effect of CR594175 silencing on expression of downstream functional protein of Wnt pathway}

We assessed E-cadherin, C-myc, CyclinD1 and MMP-9 in the ASLNC02525-depleted HepG2 cells. The results showed that C-myc, CyclinD1 and MMP-9 were decreased and E-cadherin was increased by CR594175 silencing ( $p \llbracket 0.01 \rrbracket v$ s Lv-shRNA-CR594175 group) but not the Lv-NC ( $\$ \llbracket 0.05 \rrbracket v s$ Lv-NC group) (Fig. 5). That indicated CR594175 silencing could inhibited the classic Wnt pathway by increasing the expression of CTNNB1 protein (Fig. 5).

\section{Discussion}

The invasion and metastasis of cancer refers to cancer cells break away from the primary focus and transfer to discrete tissues and proliferate into cancer of the same nature, i.e. the diffusion and growth of 
cells from primary tumor to remote organs [13]. This process depends on the interaction between cancer cells and internal environment promoting their survival, growth, and angiogenesis, as well as invasion and metastasis [14]; therefore, inhibiting proliferation and invasion of tumor cell is the key factor to inhibit tumor metastasis.

LncRNAs, endogenous RNAs longer than 200 nucleotides discovered recently and attracting much interest, have been found to have functions associated with various biological processes via epigenetic modification. Increasingly major studies suggest tangible involvement of IncRNA in cancer. Analysis of chromatin reveals that there are over 1000 IncRNAs in mammals, which are less conservative than the coding RNAs. As an important type of regulators, IncRNAs exert their functions through a variety of ways. Although they were firstly regarded as by-products by RNA polymerase II, or transcriptional noise, recent studies have shown that IncRNAs are associated with multiple biological processes such as chromosome silencing, chromatin modification and transcriptional regulation[15, 16]. Abnormal IncRNA expression is found in diversified diseases, and IncRNA expression profiles in a variety of tumors are different form normal cells. The proportion of IncRNAs in the total transcripts of genome is far larger than that of encoding RNAs. And IncRNAs play crucial roles in the regulatory network though their interaction with DNA, RNA and proteins. In addition to gene expression regulation, IncRNAs are closely related to evolution of species, embryonic development, metabolism and tumorigenesis. The evidence on involvement of IncRNAs in diseases including cancers will provide basis and target for diagnosis and treatment of diseases. Sun Shu-han et al. have found IncRNA-Dreh can inhibit hepatocellular carcinoma metastasis [17].

We screened for differential IncRNAs in several pairs of selected HCC and adjacent tissue by using IncRNA chips. The reason that CR594175 caught our attention was that its expression was not only increased in HCC than in para-carcinoma tissues, but also was increased more in HCC metastases than in primary HCC, indicating that CR594175 would be associated with the process of HCC. We knocked down CR594175 in HepG2 cells, and found that the proliferation and invasion were reduced, as well as downstream protein of Wnt pathway CMYC, CyclinD1 and MMP-9, while E-cadherin is the opposite,so we believe elevated CR594175 levels contribute to metastases of HCC through Wnt pathway. To figure out how CR594175 promotes Wnt pathway in HCC, we analyzed key proteins involved in invasion and migration in HCC cells with CR594175 silencing and control cells, and found that the expression of CTNNB1 was consistent with CR594175, which was confirmed in primary HCC, metastatic HCC and adjacent tissue. Interestingly, our RIP-Seq experiment showed that CR594175 did not bind to CTNNB1 directly (Data not shown). We next quantified the transcription and protein levels of CTNNB1 in HCC with CR594175 silencing and found that CTNNB1 expression was abnormal at the post-transcription level, suggesting an inactivation of post-regulation of CTNNB1 due to high expression of CR594175, which led to Overactivation of Wnt pathway in HCC. As a typical post-transcription regulating factor, miRNA naturally became our pointcut to investigate the relation between CR594175 and CTNNB1. Bioinformatics suggests that there is a 7-base seeding region of hsa-miR142-3p on CTNNB1's 3'UTR and 2 seeding regions on the 600-base CR594175. As a result, we speculated that elevated CR594175 bound to hsa- 
miR142-3p as miRNA sponge and disabled the negative regulation of CTNNB1 by hsa-miR142-3p, so CTNNB1 expression was increased and resulted in proliferation and invasion of HCC cells.

The interaction between IncRNAs and miRNAs has an important influence on the onset and development of cancer $[17,18]$. MiRNAs are able to regulate IncRNAs in a targeted way: a study has shown that miR-21 targets IncRNA GAS5 in addition to protein coding genes [19]. LncRNAs can also affect the onset and development of cancer by regulating expression of miRNAs. According to existing studies, IncRNAs regulate miRNAs through three ways: (1) to competitively combine to 3'-UTR of mRNAs with miRNAs, so as to inhibit negative regulation by miRNAs-Pang et al. found that an anti-sense RNA complementary to BACE1 can complement to BACE1 mRNA, competitively inhibiting the negative regulation of BACE1 by miRNA[20]; (2) to regulate target genes by forming pre-miRNAs after RNA splicing and producing specific miRNAs; and (3) to act as endogenous miRNA sponge to suppress miRNA function, so as to affect malignant biological behavior of cancer cells[21]. The most important finding of this study is CR594175 silencing could restore the negative regulation of CTNNB1 by hsa-miR142-3p to inhibit cancer, directly based on following facts: (1) hsa-miR142-3p negatively regulated CTNNB1 by binding to its 3'UTR, which was found in HCC with CR594175 silencing but not with those with high CR594175 expression levels; (2)CR594175 silencing inhibited proliferation and invasion of HCC cells, which was reversed by overexpression of CTNNB1; (3) Overexpression of hsa-miR142-3p had no observable effect on proliferation and invasion of HCC cells, but inhibited that when CR594175 was depleted. Considering CTNNB1 overexpressed by the lentiviral system has no wild 3'UTR, it would not be affected by miRNA. So we think that there is a CR594175/hsa-miR142-3p/CTNNB1 pathway regulating metastasis of HCC.

\section{Conclusion}

The study demonstrates that CR594175 plays a key role in the process of HCC metastasis, and offers a possible explanation for that hsa-miR142-3p loses its basic function of resisting HCC tumors. In the long run, IncRNA will not only be a direct target for gene therapy but also can be used together with miRNA for better effect.

\section{Abbreviations}

1. HCC Hepatocellular carcinoma

2. CTNNB1 Catenin, beta-1

3. UTR Untranslated Regions

4. FBS Fetal bovine calf serum

5. DMEM Dulbecco minimum essential medium

6. NC negative control

7. bp base-pair

8. LSD Least Significant Difference 


\section{Declarations}

Ethics approval and consent to participate

Not applicable.

\section{Consent for publication}

Not applicable.

\section{Availability of data and materials}

All data generated or analyzed during this study are included in this published article [and its supplementary information files].

\section{Competing interests}

The authors declare that they have no competing interests.

\section{Funding}

Not applicable.

\section{Authors contributions}

$L Q$ and $Y X X$ performed the data curation, methodology, project administration and wrote original draft.YMJ,LXK,ZXJ,LYJ,CZ,FQX,LWC collected data and performed statistical analysis.SLJ and LWC reviewed and edited the manuscript.All authors read and approved the final manuscript.

\section{Acknowledgements}

Not applicable.

\section{References}

[1] Mulcahy MF. Management of hepatocellular cancer. Curr Treat Options Oncol, 2005, 6(5):423-435.

[2] Bosch FX, Ribes J, Diaz M, et al. Primary liver cancer: worldwide incidence and trends. Gastroenterology, 2004, 127:S5-S16. 
[3] El-Serag HB. Hepatocellular carcinoma. N Engl J Med, 2011, 365(12):1118-1127.

[4] Yang $\mathrm{H}$, Lin $\mathrm{M}$, Xiong $\mathrm{F}$, et al. Combined lysosomal protein transmembrane 4 beta-35 and argininosuccinate synthetase expression predicts clinical outcome in hepatocellular carcinoma patients.Surg Today, 2011, 41(6):810-817.

[5] Abdelmohsen K, Gorospe M. Noncoding RNA control of cellular enescence. Wiley Interdiscip Rev RNA. 2015;6(6):615-29.

[6] Alaei-Mahabadi B, Larsson E. Limited evidence for evolutionarily conserved targeting of long noncoding RNAs by microRNAs. Silence. 2013;4(1):4.

[7] Schmitt AM, Chang HY.Long Noncoding RNAs in Cancer Pathways. Cancer Cell. 2016;29(4):452-63.

[8]Behari J, Zeng G, Otruba W, et al. R-Etodolac decreases $\beta$-catenin levels along with survival and proliferation of hepatoma cells. Hepatology, 2007, 46: 849-857.

[9]Loeppen S, Koehle C, Buchmann A, et al. A beta-catenin-de-pendent pathway regulates expression of cytochrome P450 iso-forms in mouse liver tumors. Carcinogenesis, 2005, 26:239-248.

[10]Yang C, Xu Y, Cheng F, Hu Y, Yang S, Rao J, et al. miR-1301 inhibits hepatocellular carcinoma cell migration, invasion, and angiogenesis by decreasing Wnt/ $\beta$-catenin signaling through targeting BCL9. Cell Death Dis. 2017;8(8):e2999.

[11]Jin Y, Wang J, Han J, Luo D, Sun Z. MiR-122 inhibits epithelial-mesenchymal transition in hepatocellular carcinoma by targeting CTNNB1 and Snail2 and suppressing WNT/ $\beta$-cadherin signaling pathway. Exp Cell Res. 2017;360(2):210-217.

[12]Ashmawy AM, Elgeshy KM, Abdel Salam ET, Ghareeb M, Kobaisi MH, Amin HAA, et.al. Crosstalk between liver-related microRNAs and Wnt/ $\beta$-catenin pathway in hepatocellular carcinoma patients. Arab $J$ Gastroenterol. 2017;18(3):144-150.

[13]Cui H, Song R, Wu J, Wang W, Chen X, Yin J. MicroRNA-337 regulates the PI3K/AKT and Wnt/ $\beta$ catenin signaling pathways to inhibit hepatocellular carcinoma progression by targeting high-mobility group AT-hook 2. Am J Cancer Res. 2018;8(3):405-421. eCollection 2018.

[14]Ponting CP, Oliver PL, Reik W. Evolution and functions of long noncoding RNAs. Cell 2009; 136: 629 $-641$

[15]Bergmann JH, Spector DL. Long non-coding RNAs: modulators of nuclear structure and function. Curr Opin Cell Biol. 2014;26:10-8.

[16]Deng G, Sui G. Noncoding RNA in oncogenesis: a new era of identifying key players. Int J Mol Sci. 2013;14(9):18319-49. 
[17]Pickard MR, Williams GT. Molecular and Cellular Mechanisms of Action of Tumour Suppressor GAS5 LncRNA. Genes (Basel). 2015;6(3):484-99.

[18]Pang M, Xing C, Adams N, et al. Comparative expression of miRNA genes and miRNA-based AFLP marker analysis in cultivated tetraploid cottons. J Plant Physiol. 2011;168(8):824-30.

[19]Brodersen P, Sakvarelidze-Achard L, Schaller H, et.al. Isoprenoid biosynthesis is required for miRNA function and affects membrane association of ARGONAUTE 1 in Arabidopsis. Proc Natl Acad Sci U S A. 2012;109(5):1778-83.

[20]Huang JF, Guo YJ, Zhao CX, et al. Hepatitis B virus X protein ( $\mathrm{HBx}$ )-related long noncoding RNA (IncRNA) down-regulated expression by HBx (Dreh) inhibits hepatocellular carcinoma metastasis by targeting the intermediate filament protein vimentin. Hepatology. 2013;57(5):1882-92.

[21]Lai MC, Yang Z, Zhou L, et al. Long non-coding RNA MALAT-1 overexpression predicts tumor recurrence of hepatocellular carcinoma after liver transplantation. Med Oncol. 2012;29(3):1810-6.

\section{Table 1}

Tab 1 Clinicopathological features of 24 patients with metastatic HCC

\begin{tabular}{|c|c|c|c|c|}
\hline Mumber & Gender & Age & TNM stage & Metastasis Type \\
\hline 1 & $\mathrm{~F}$ & 55 & T2N1M1 & Lung \\
\hline 2 & M & 62 & T3N1M1 & Lung \\
\hline 3 & $\mathrm{~F}$ & 61 & T2NXM1 & Stomach \\
\hline 4 & $\mathrm{~F}$ & 55 & T2N0M1 & Head \\
\hline 5 & $\mathrm{~F}$ & 58 & T3NXM1 & Colon \\
\hline 6 & M & 66 & T3N0M1 & Lung \\
\hline 7 & M & 73 & T2N1M1 & Stomach \\
\hline 8 & $\mathrm{~F}$ & 55 & T3N1M1 & Colon \\
\hline 9 & M & 42 & T2N1M1 & Stomach \\
\hline 10 & $\mathrm{~F}$ & 58 & T3N0M1 & Lung \\
\hline 11 & $\mathrm{M}$ & 51 & T3N1M1 & Stomach \\
\hline 12 & M & 44 & T2N0M1 & Stomach \\
\hline 13 & $\mathrm{~F}$ & 53 & T3NXM1 & Lung \\
\hline 14 & $\mathrm{~F}$ & 66 & T3N1M1 & Colon \\
\hline 15 & M & 62 & T3N0M1 & Stomach \\
\hline 16 & $\mathrm{M}$ & 60 & T2NXM1 & Lung \\
\hline 17 & $\mathrm{~F}$ & 52 & T3N1M1 & Lung \\
\hline 18 & $\mathrm{~F}$ & 47 & T3N0M1 & Lung \\
\hline 19 & M & 52 & T2N0M1 & Bone \\
\hline 20 & $\mathrm{~F}$ & 58 & T2N0M1 & Stomach \\
\hline 21 & M & 69 & T3NXM1 & Lung \\
\hline 22 & $\mathrm{~F}$ & 70 & T2NXM1 & Lung \\
\hline 23 & $\mathrm{~F}$ & 42 & $\mathrm{~T} 4 \mathrm{~N} 1 \mathrm{M} 1$ & Colon \\
\hline 24 & M & 53 & T3N1M1 & Bone \\
\hline
\end{tabular}


Figures
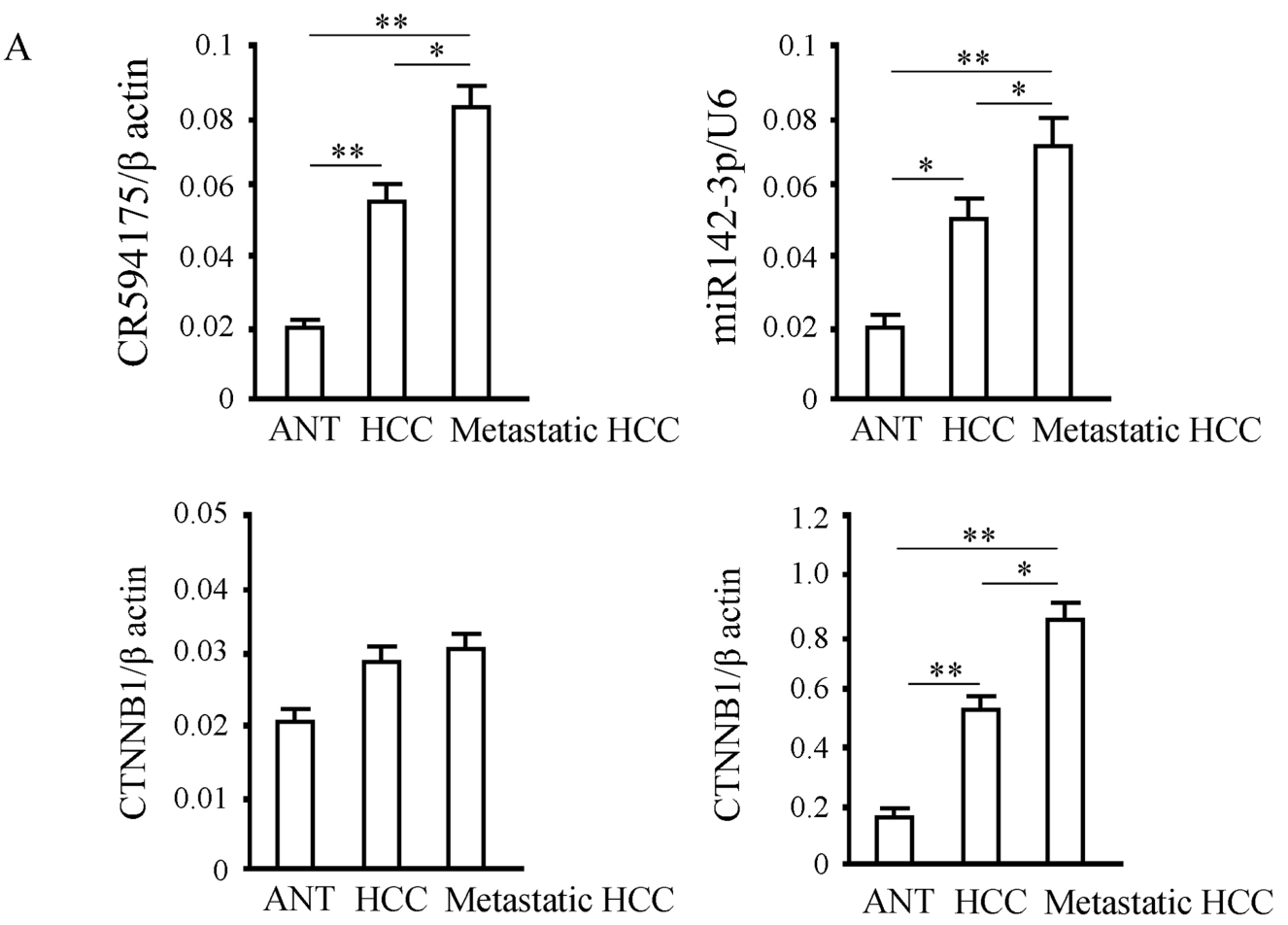

ANT HCC Metastatic HCC

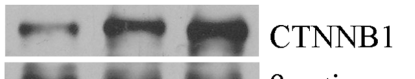

O5 $\beta$ actin
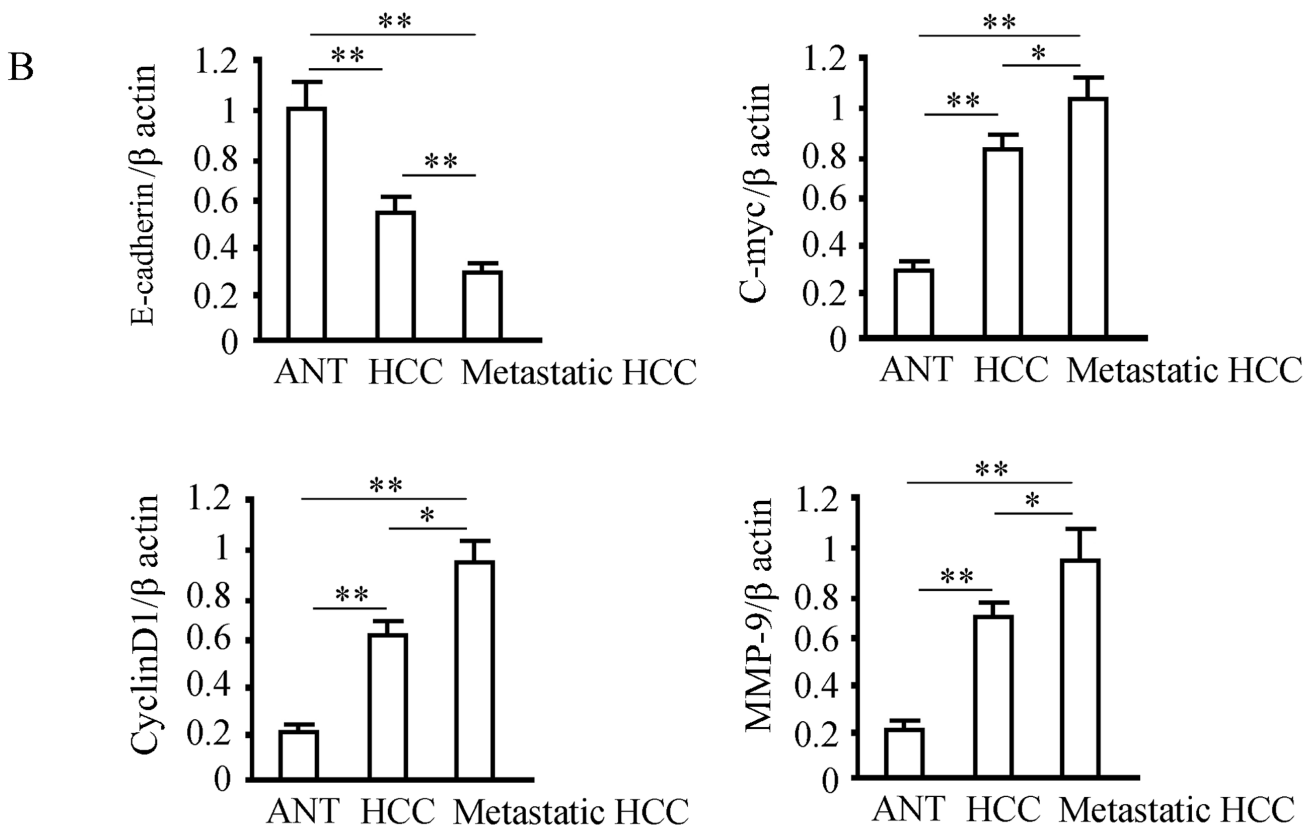

ANT HCC Metastatic HCC

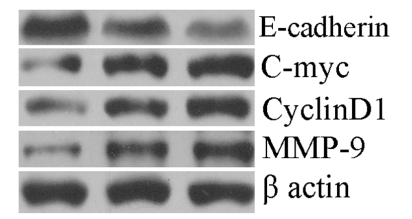

Figure 1 
Detection of IncRNA-CR594175,hsa-miR142-3p,CTNNB1 and proteins related to Wnt pathway in the normal adjacent tissue, the HCC tumors and metastatic tissues. A)CR594175,hsa-miR142-3p, CTNNB1 mRNA and protein levels in normal adjacent tissues, HCC tissues and metastatic tissue measured by Real Time PCR or western blotting. B)Protein levels of E-cadherin(95 kDa), C-myc(56 kDa), CyclinD1(36 kDa) and MMP-9(102 kDa) in the normal adjacent tissues, HCC tissues and metastatic tissue measured by western blotting: upper: representative blots; lower: the optical density of the target band divided by the optical density of the $\beta$-actin band; Data are expressed as mean \pm SD. $*, p<0.05$ and $* *, p<0.01$, t-test.

A
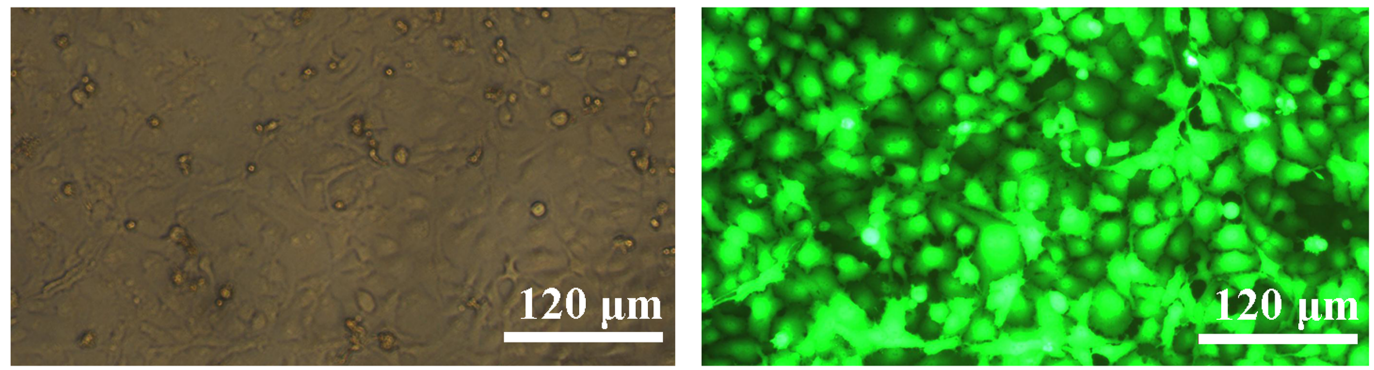

Cell infected with Lv-CR594175 (MOI=10) 72hrs later
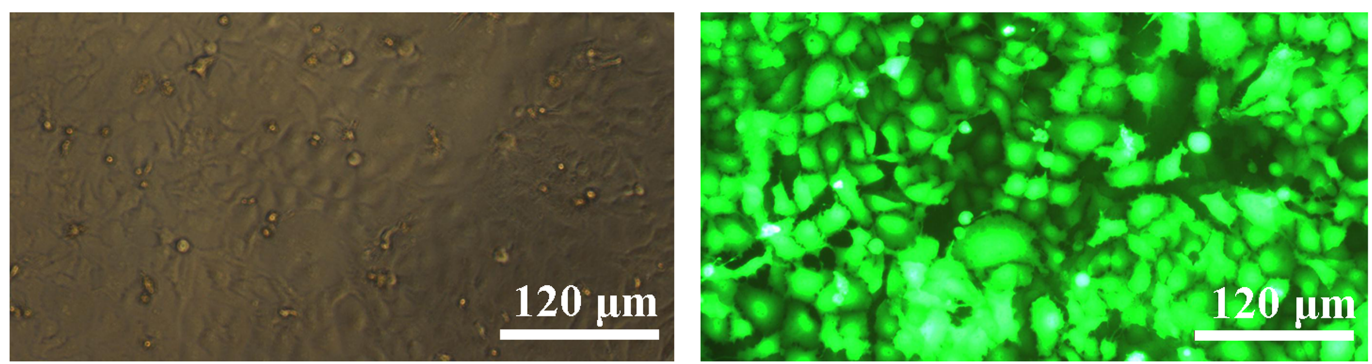

Cell infected with Lv-CTNNB1 (MOI=10) 72hrs later

$\mathrm{B}$
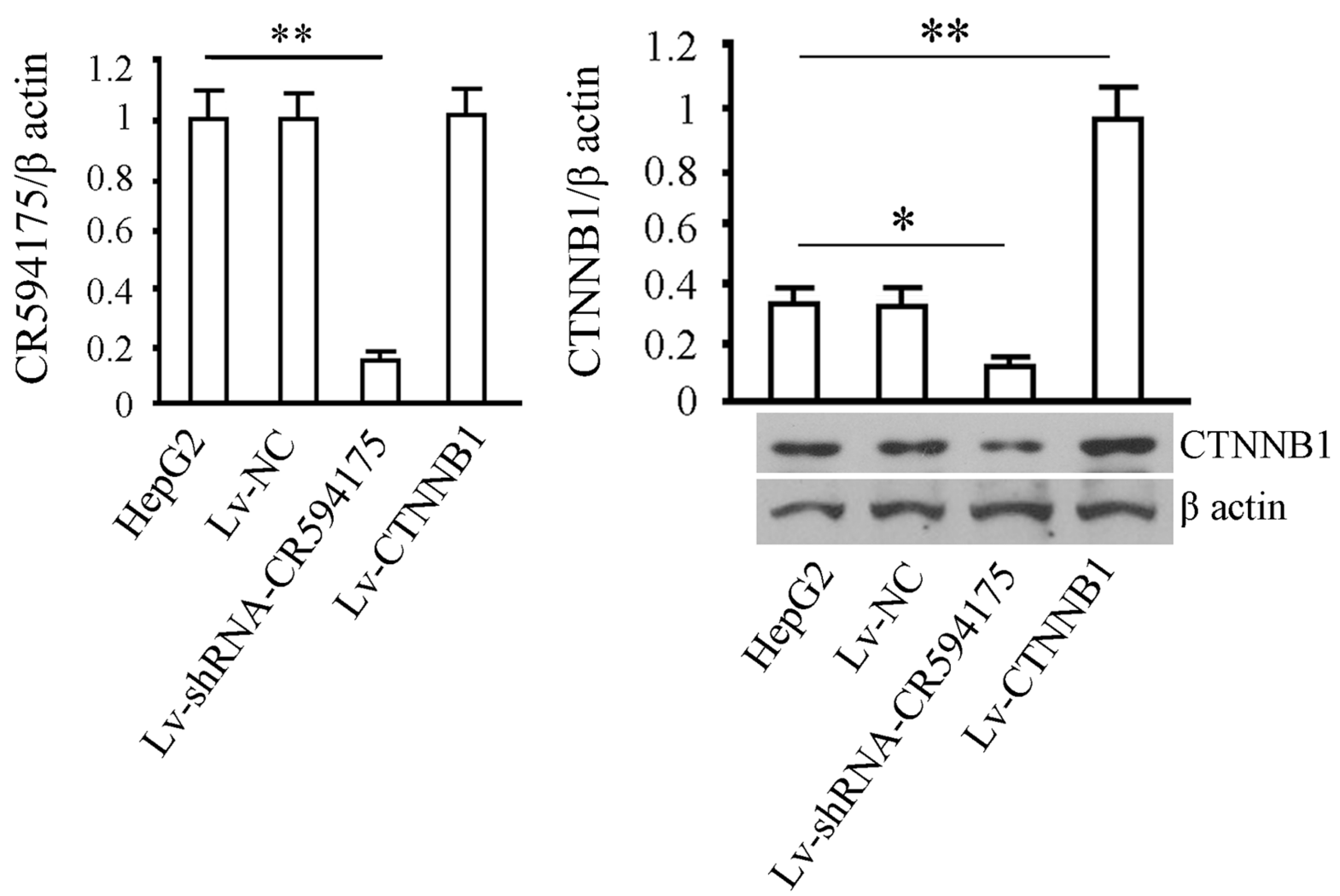
Figure 2

Genetic intervention through a lentiviral approach A)GFP expression 72 hours after HepG2 was infected with recombinant virus. The infection rate was estimated by dividing the number of the cells expressing GFP with the number of all the cells in each view. For statistics, five views were randomly selected, and the mean was calculated (Fig2.A); B)Determination of CR594175 and CTNNB1 protein. For realtime-PCR and western blotting, $\beta$ actin served as the internal reference. ${ }^{\star \star} p<0.01$, vs cell group. The tests were carried out on three biological triplicates, and data are expressed as the mean \pm SD.

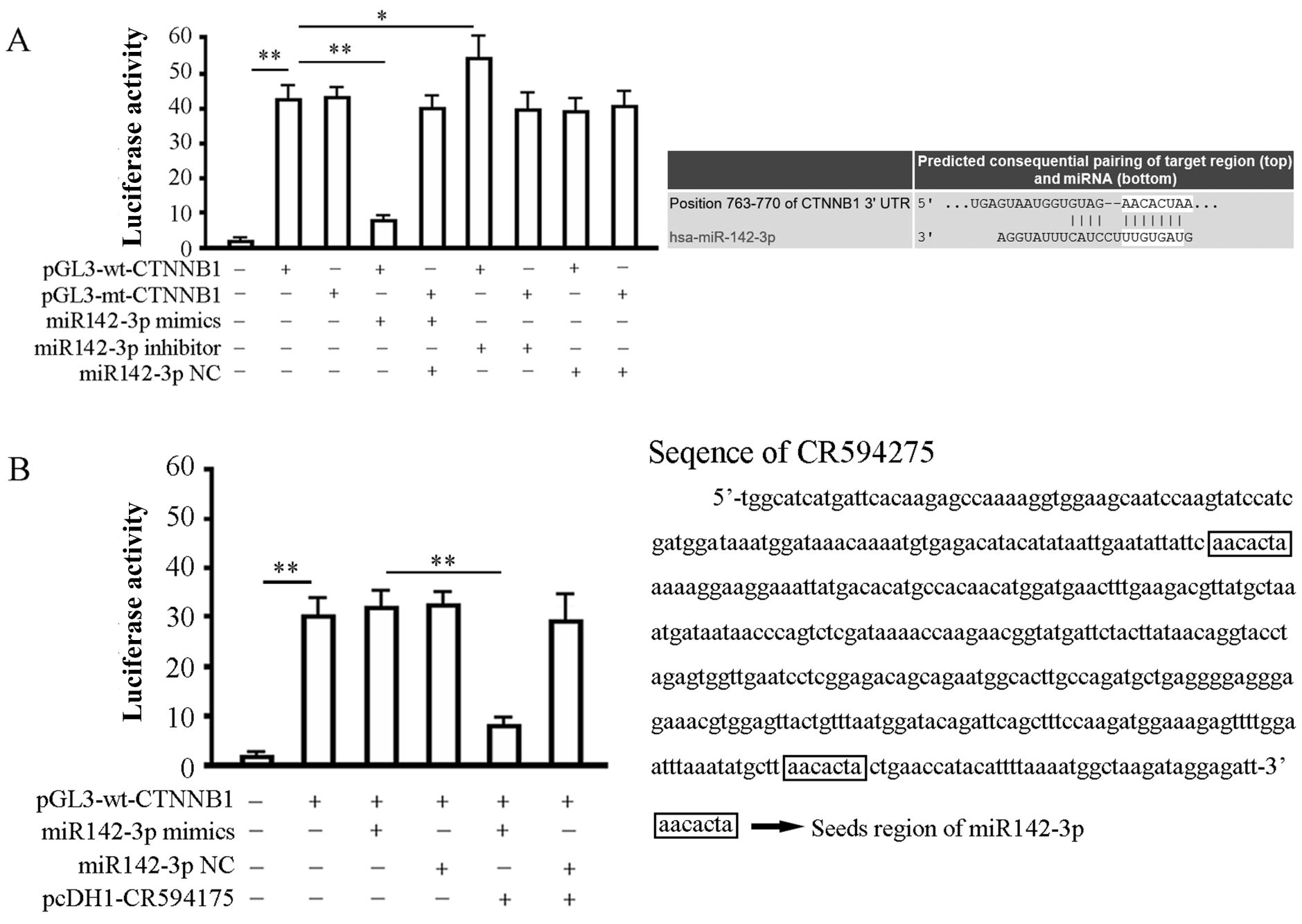

\section{Figure 3}

Hsa-miR142-3p binds to CTNNB1 3'UTR, which is interfered by CR594175. A)293 cells were transfected with pGL3-wt-CTNNB1 or pGL3-mt-CTNNB1 in the presence or absence of miR142-3p-mimic or inhibitor and subjected to luciferase activity assay 48 hours later. Uper panel, predicted binding site of hsamiR142-3p in 3'-UTR of CTNNB1; Lower panel, Effects of hsa-miR142-3p on the expression of a luciferase cassette encoding the CTNNB1 3'-UTR. The histogram shows the relative firefly luciferase activity for the different experimental groups. ${ }^{*}, p<0.05$, and $* *, p<0.01$, compared with the group transfected with the same vector but without the miR142-3p mimics or miR142-3p inhibitor. B)Left panel, HepG2 cells were transfected with the indicated vectors and subjected to luciferase activity assay 48 hours later. Uper 
panel, predicted binding site of hsa-miR142-3p in CR594175; Right panel, expression of a luciferase cassette encoding the CR594175. The histogram shows the relative firefly luciferase activity for the different experimental groups. ${ }^{*}, \mathrm{p}<0.05$, compared with the group transfected pGL3-wt-CTNNB1 and miR142-3p-mimics. Data are expressed as mean \pm SD of at least three independent experiments.

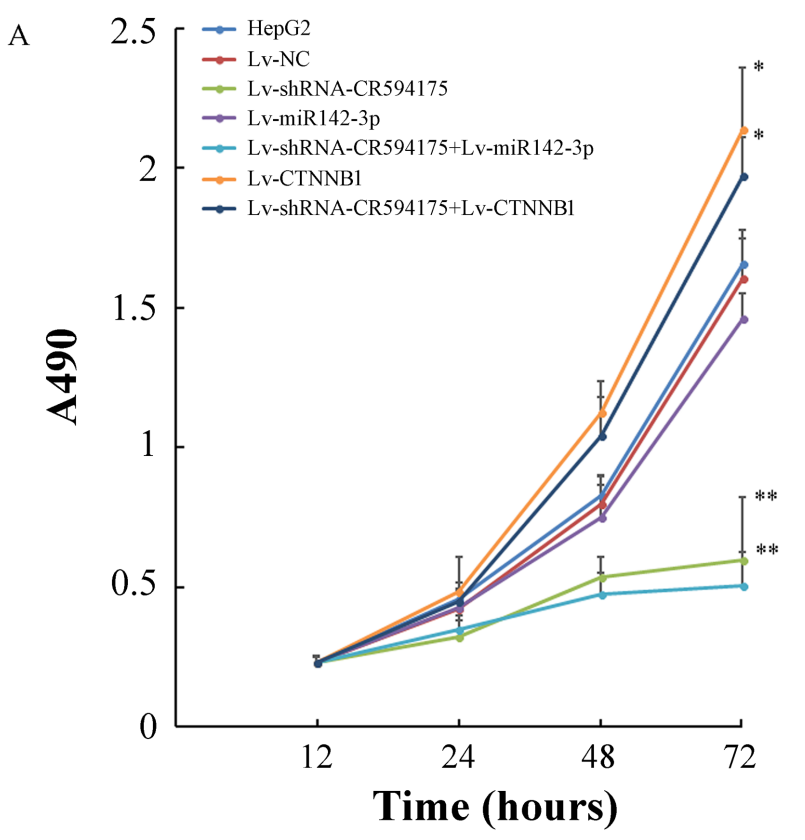

B
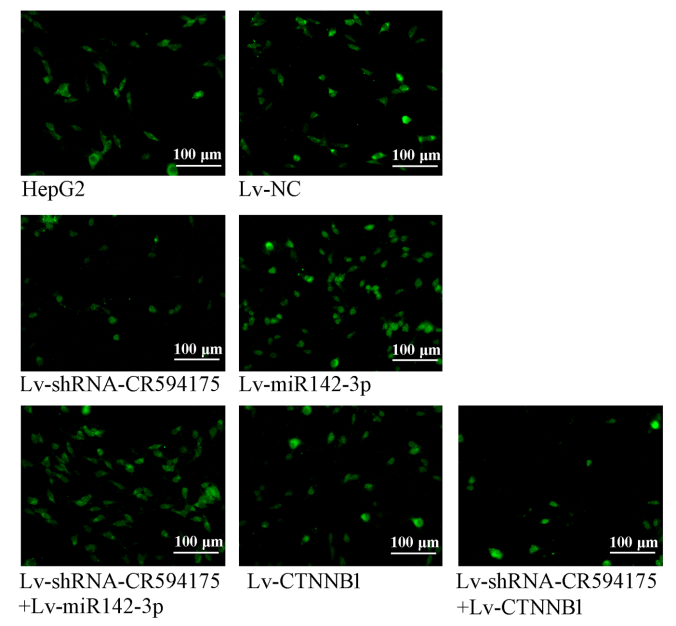

C

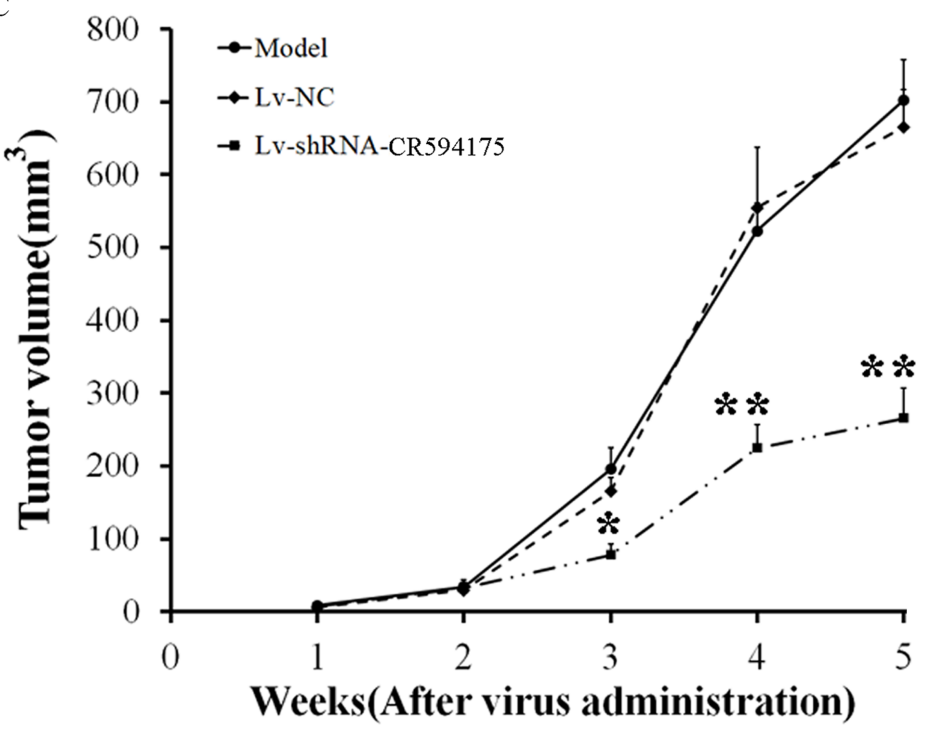

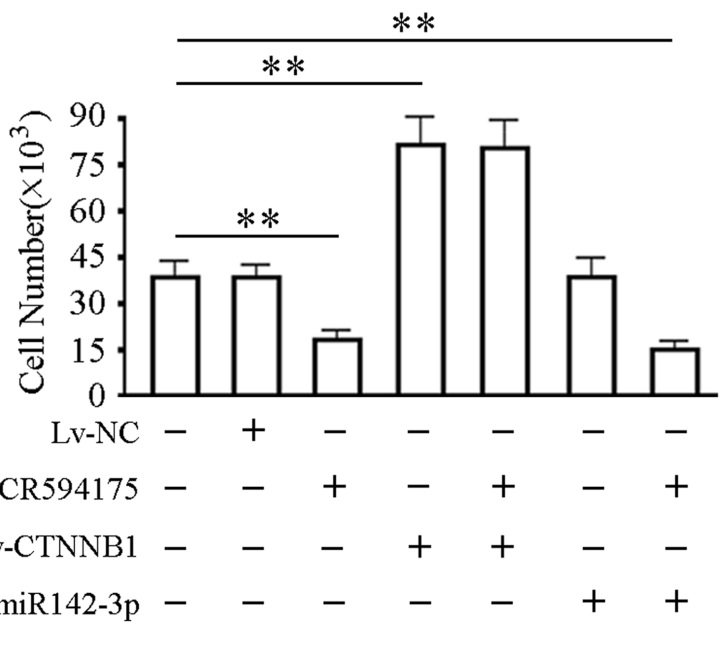

Figure 4

Effects of CR594175 depletion on proliferation and invasion of HCC cells and In vivo tumor suppression A)HepG2 were infected with the indicated lentivirus and then seeded to 96-well plates and subjected to cell vitality assay at the $0,24,48$ and 72 hours. B) Cell invasion assay 48 hours after HepG2 infected with the indicated lentivirus seeding into the upper chamber of a transwell. Upper, representative photos of cells which passed through the basement membrane, lower, statistics of the numbers of invasive cells. C) Growth curves of tumor in vivo. The x-coordinate represents the period of injection and the y-coordinate 
represents the tumor volume. The formula for calculating the tumor volume was: $V=a b 2 / 2$, where $a$ and $b$ are the long and short diameters of the tumor. The number of animals in one group was $12(n=12)$. ** $p<0.01$, ${ }^{*}<0.05$ vs CR594175 silencing group, data are expressed as the means \pm SD.
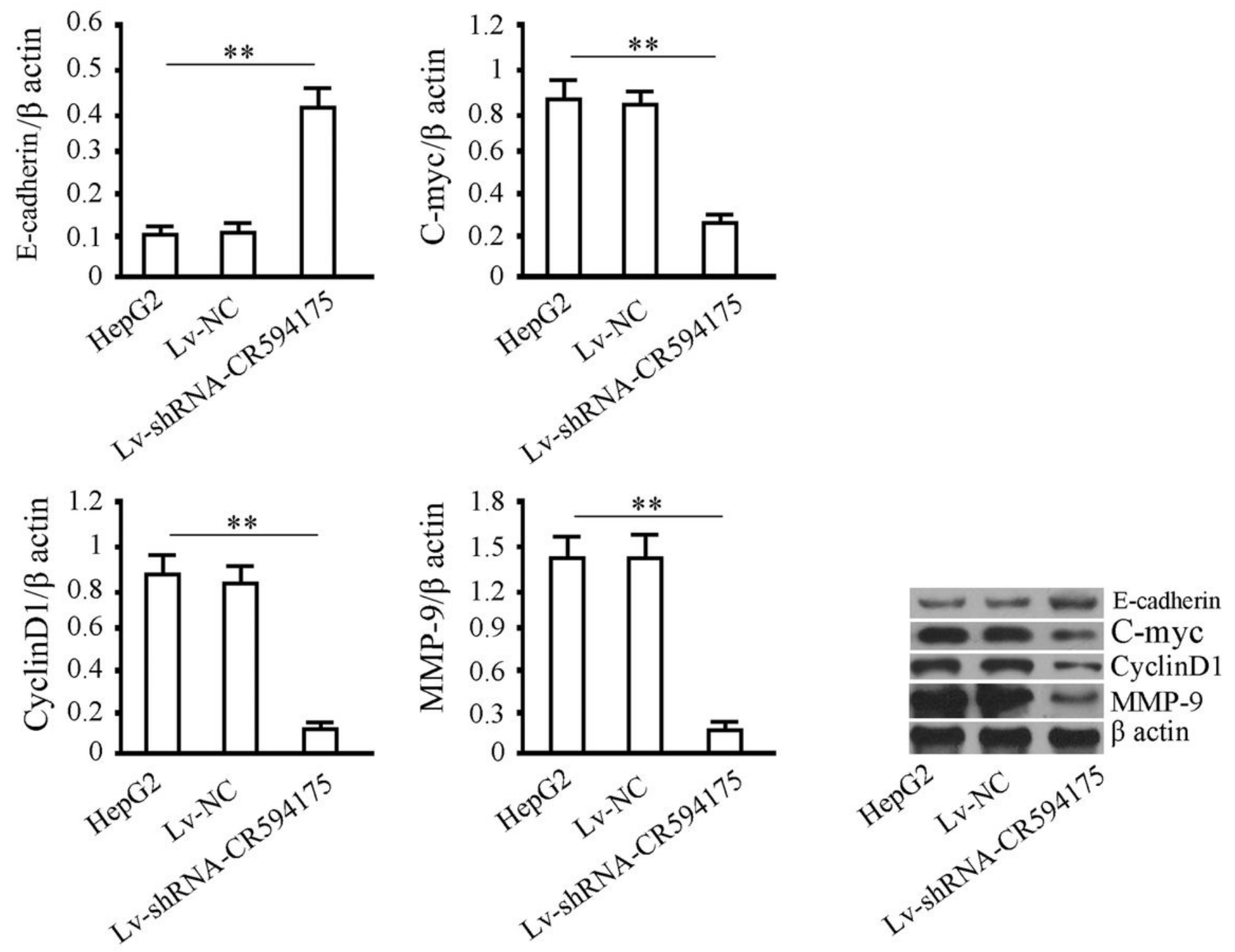

\section{Figure 5}

Effects of CR594175 silencing on E-cadherin, C-myc, CyclinD1 and MMP-9 HepG2 infected with or without Lv-NC or Lv-shRNA-CR594175 72 hours post infection were detected using western blot. $\beta$ actin $(43 \mathrm{kDa})$ as a loading control. Data were representative of at least three independent experiments. ** $p<0.01,{ }^{*} p<0.05 . t$ test. 